TRANSACTIONS OF THE

AMERICAN MATHEMATICAL SOCIETY

Volume 363, Number 8, August 2011, Pages 4263-4283

S 0002-9947(2011)05217-7

Article electronically published on March 3, 2011

\title{
SYMMETRIC ROOTS AND ADMISSIBLE PAIRING
}

\author{
ROBIN DE JONG
}

\begin{abstract}
Using the discriminant modular form and the Noether formula it is possible to write the admissible self-intersection of the relative dualising sheaf of a semistable hyperelliptic curve over a number field or function field as a sum, over all places, of a certain adelic invariant $\chi$. We provide a simple geometric interpretation for this invariant $\chi$, based on the arithmetic of symmetric roots. We propose the conjecture that the invariant $\chi$ coincides with the invariant $\varphi$ introduced in a recent paper by S.-W. Zhang. This conjecture is true in the genus 2 case, and we obtain a new proof of the Bogomolov conjecture for curves of genus 2 over number fields.
\end{abstract}

\section{INTRODUCTION}

Let $X$ be a hyperelliptic curve of genus $g \geq 2$ over a field $K$ which is either a number field or the function field of a curve over a field. Assume that $X$ has semistable reduction over $K$. We study, for each place $v$ of $K$, a real-valued invariant $\chi\left(X_{v}\right)$ of $X \otimes K_{v}$, with the following two properties:

(i) $\chi\left(X_{v}\right)=0$ if $v$ is non-Archimedean and $X$ has good reduction at $v$;

(ii) for the admissible self-intersection of the relative dualising sheaf $(\omega, \omega)_{a}$ of $X$ the formula

$$
(\omega, \omega)_{a}=\frac{2 g-2}{2 g+1} \sum_{v} \chi\left(X_{v}\right) \log N v
$$

holds. Here $v$ runs over the places of $K$, and the $N v$ are usual local factors related to the product formula for $K$.

In the function field context, the invariant $\chi$ already appears in work of A. Moriwaki $\left[13\right.$ and K. Yamaki [16], albeit in disguise. It follows from their work that $\chi\left(X_{v}\right)$ is strictly positive if $X$ has non-smooth reduction at $v$. In fact they prove a precise lower bound for $\chi\left(X_{v}\right)$ in terms of the geometry of the special fiber at $v$. If $X$ has a non-isotrivial model, by property (ii) this yields as a corollary an effective proof of the Bogomolov conjecture for $X$, i.e. the strict positivity of $(\omega, \omega)_{a}$.

The precise definition of $\chi$ is given in Section 4. It involves the discriminant modular form of weight $8 g+4$, suitably normalised, the $\varepsilon$-invariant of S.-W. Zhang [18, and the $\delta$-invariant appearing in the Noether formula for smooth projective curves over $K$.

Received by the editors June 29, 2009 and, in revised form, October 5, 2009.

2010 Mathematics Subject Classification. Primary 11G20, 14G40.

Key words and phrases. Hyperelliptic curves, local fields, admissible pairing, self-intersection of the relative dualising sheaf, symmetric roots.

(C)2011 American Mathematical Society Reverts to public domain 28 years from publication 
Our purpose is to give a geometric interpretation of the invariant $\chi$. Fix, for each place $v$ of $K$, an algebraic closure $\bar{K}_{v}$ of $K_{v}$. Endow each $\bar{K}_{v}$ with a standard absolute value $|\cdot|_{v}$ (see Section 3 ). Then we prove:

Theorem A. Let $\langle\omega, \omega\rangle$ be the Deligne self-pairing of the dualising sheaf $\omega$ of $X$ on $\operatorname{Spec}(K)$. There exists a canonical section $q$ of $(2 g+1)\langle\omega, \omega\rangle$ on $\operatorname{Spec}(K)$, obtained by pullback from the moduli stack of smooth hyperelliptic curves of genus $g$ over $\mathbb{Z}$, such that the equality

$$
-\log |q|_{a}=(2 g-2) \chi\left(X_{v}\right)
$$

holds for each place $v$ of $K$. Here $|\cdot|_{a}$ is Zhang's admissible norm on $(2 g+1)\langle\omega, \omega\rangle$ at $v$.

The construction of $q$ yields the following simple formula for $\chi$.

Theorem B. Assume that $K$ does not have characteristic 2. Let $v$ be a place of $K$, and let $w_{1}, \ldots, w_{2 g+2}$ on $X \otimes \bar{K}_{v}$ be the Weierstrass points of $X \otimes \bar{K}_{v}$. Then for each $i=1, \ldots, 2 g+2$ the formula

$$
\chi\left(X_{v}\right)=-2 g\left(\log |2|_{v}+\sum_{k \neq i}\left(w_{i}, w_{k}\right)_{a}\right)
$$

holds, where ()$_{a}$ is Zhang's admissible pairing on $\operatorname{Div}\left(X \otimes \bar{K}_{v}\right)$.

In a recent paper [18 S.-W. Zhang introduced, for any smooth projective geometrically connected curve $X$ of genus at least 2 over $K$, an invariant $\varphi\left(X_{v}\right)$ for each $X \otimes K_{v}$ such that property (i) holds for $\varphi$, and property (ii) holds for $\varphi$ if $X$ is hyperelliptic. We propose the conjecture that $\varphi$ and $\chi$ are equal for all hyperelliptic curves over $K$ and all places of $K$. This conjecture turns out to be true in the case $g=2$. As we will explain below, this gives a new proof of the Bogomolov conjecture for curves of genus 2 over number fields.

The main tools in this paper are moduli of (pointed) stable hyperelliptic curves and the arithmetic of symmetric roots of $X$. These symmetric roots were extensively studied by J. Guàrdia [4] in the context of an effective Torelli theorem for hyperelliptic period matrices. They are defined as follows. Let $\kappa$ be any field of characteristic not equal to 2 and let $X$ be a hyperelliptic curve of genus $g \geq 2$ over $\kappa$. Fix a separable algebraic closure $\bar{\kappa}$ of $\kappa$. Let $w_{1}, \ldots, w_{2 g+2}$ on $X \otimes \bar{\kappa}$ be the Weierstrass points of $X \otimes \bar{\kappa}$, and let $h: X \rightarrow \mathbb{P}_{\kappa}^{1}$ be the quotient under the hyperelliptic involution of $X$. Fix a coordinate $x$ on $\mathbb{P}_{\bar{\kappa}}^{1}$, and suppose that $w_{i}$ gets mapped to $a_{i}$ on $\mathbb{P}_{\bar{\kappa}}^{1}$. Take a pair $\left(w_{i}, w_{j}\right)$ of distinct Weierstrass points, and let $\tau$ be an automorphism of $\mathbb{P}_{\bar{\kappa}}^{1}$ such that $\tau\left(a_{i}\right)=0, \tau\left(a_{j}\right)=\infty$, and $\prod_{k \neq i, j} \tau\left(a_{k}\right)=1$. The $\tau\left(a_{k}\right)$ for $k \neq i, j$ are finite, non-zero, and well-defined up to a common scalar from $\mu_{2 g}$, the set of $2 g$-th roots of unity in $\bar{\kappa}$. The resulting subset of $S_{2 g} \backslash \mathbb{A}_{\bar{\kappa}}^{2 g} / \mu_{2 g}$ is denoted by $\left\{\ell_{i j k}\right\}_{k \neq i, j}$ and is called the set of symmetric roots on $X$ and $\left(w_{i}, w_{j}\right)$. This is clearly an invariant of $X$ and the pair $\left(w_{i}, w_{j}\right)$ over $\bar{\kappa}$. It is easily checked that the formula

$$
\ell_{i j k}=\frac{a_{i}-a_{k}}{a_{j}-a_{k}} \sqrt[2 g]{\prod_{r \neq i, j} \frac{\left(a_{j}-a_{r}\right)}{\left(a_{i}-a_{r}\right)}}
$$

holds for each $k \neq i, j$. This formula of course has to be interpreted appropriately if one of the $a_{i}, a_{j}, a_{k}$ equals infinity. For each given $k \neq i, j$, the element $\ell_{i j k}^{2 g}$ of $\bar{\kappa}$ lies in the field of definition inside $\bar{\kappa}$ of the triple $\left(w_{i}, w_{j}, w_{k}\right)$. 
Our main result, Theorem 3.1, gives a description of a power of $\ell_{i j k}^{2 g}$ as a rational function on the moduli stack of hyperelliptic curves with three marked Weierstrass points. As a corollary of this result we obtain the following remarkable formula, expressing the norm of a symmetric root as a special value of Zhang's admissible pairing on divisors.

Theorem C. Assume that $K$ does not have characteristic 2. Let $v$ be a place of $K$, and let $w_{i}, w_{j}, w_{k}$ be three distinct Weierstrass points on $X \otimes \bar{K}_{v}$. Let $\ell_{i j k}$ in $\bar{K}_{v}^{\times} / \mu_{2 g}$ be the symmetric root on the triple $\left(w_{i}, w_{j}, w_{k}\right)$. Then the formula

$$
\left(w_{i}-w_{j}, w_{k}\right)_{a}=-\frac{1}{2} \log \left|\ell_{i j k}\right|_{v}
$$

holds.

We mention that for an Archimedean place $v$ this result says that

$$
\frac{G_{v}\left(w_{i}, w_{k}\right)}{G_{v}\left(w_{j}, w_{k}\right)}=\sqrt{\left|\ell_{i j k}\right|_{v}},
$$

where $G_{v}$ is the Arakelov-Green's function on the compact Riemann surface $X\left(\bar{K}_{v}\right)$. This formula is remarkable since it says that a special value of some transcendental function on $X\left(\bar{K}_{v}\right)$ is algebraic. We believe that this fact merits further attention.

The main result and Theorem $\mathrm{C}$ are proven in Section 3 . After introducing the $\chi$-invariant in Section 4 we give in Section 5 our more intrinsic approach to $\chi$ and prove Theorems A and B. In Section [6 we compare $\chi$ with Zhang's $\varphi$-invariant. Throughout, the reader is assumed to be familiar with the theory of admissible pairing on curves as in [17]. All schemes and algebraic stacks in this paper are assumed locally Noetherian and separated.

\section{AdMissible PAIRING AND RELATIVE DUALISING SHEAF}

We begin with a useful description of $\langle\omega, \omega\rangle$ for families of semistable hyperelliptic curves. The contents of this section straightforwardly generalise those of [2, Section 1] which treats the genus 2 case.

We start with a few definitions. Let $S$ be a (locally Noetherian, separated) scheme. A proper flat family $\pi: X \rightarrow S$ of curves of genus $g \geq 2$ is called a smooth hyperelliptic curve over $S$ if $\pi$ is smooth and admits an involution $\sigma \in \operatorname{Aut}_{S}(X)$ such that $\sigma$ restricts to a hyperelliptic involution in each geometric fiber of $\pi$. If $\pi$ is a smooth hyperelliptic curve, the involution $\sigma$ is uniquely determined. We call $\pi: X \rightarrow S$ a generically smooth semistable hyperelliptic curve if $\pi$ is semistable, and there exist an open dense subscheme $U$ of $S$ and an involution $\sigma \in \operatorname{Aut}_{S}(X)$ such that $X_{U}$ together with the restriction of $\sigma$ to $X_{U}$ is a smooth hyperelliptic curve over $U$. Again, if $\pi$ is a generically smooth semistable hyperelliptic curve, the involution $\sigma$ is unique; we call $\sigma$ the hyperelliptic involution of $X$ over $S$.

Let $\pi: X \rightarrow S$ be a generically smooth semistable hyperelliptic curve of genus $g \geq 2$. Let $\omega$ be the relative dualising sheaf of $\pi$ and let $W$ be a $\sigma$-invariant section of $\pi$ with image in the smooth locus of $\pi$. The image of $W$ in $X$ induces a relative Cartier divisor on $X$ which we also denote by $W$. We make the convention that whenever a Cartier divisor on a scheme is given, the associated line bundle will be denoted by the same symbol. Moreover we use additive notation for the tensor product of line bundles. 
Assume for the moment that $\pi$ is smooth. By [5, Lemma 6.2] there exists a unique isomorphism:

$$
\omega \stackrel{\cong}{\rightrightarrows}(2 g-2) W-(2 g-1) \pi^{*}\langle W, W\rangle,
$$

compatible with base change, such that pullback along $W$ induces the adjunction isomorphism:

$$
\langle W, \omega\rangle \stackrel{\cong}{\rightarrow}\langle W, W\rangle
$$

on $S$. Here $\langle\cdot, \cdot\rangle$ denotes Deligne pairing of line bundles on $X$. Now drop the condition that $\pi$ is smooth. By the above isomorphisms we have a canonical nonzero rational section $s$ of the line bundle:

$$
\omega-(2 g-2) W+(2 g-1) \pi^{*}\langle W, W\rangle
$$

on $X$. Denote by $V$ its divisor on $X$; then $V$ is disjoint from the smooth fibers of $\pi$, and $W^{*} V=\langle W, V\rangle$ is canonically trivial on $S$.

Next let $\left(W_{i}, W_{k}\right)$ be a pair of $\sigma$-invariant sections of $\pi$ with image in the smooth locus of $\pi$. Denote by $V_{i}, V_{k}$ the associated Cartier divisors supported in the nonsmooth fibers of $\pi$. We define a line bundle $Q_{i k}$ on $S$ associated to $\left(W_{i}, W_{k}\right)$ as follows:

$$
Q_{i k}=-4 g(g-1)\left\langle W_{i}, W_{k}\right\rangle-\left\langle W_{i}, V_{k}\right\rangle-\left\langle V_{i}, W_{k}\right\rangle+\left\langle V_{i}, V_{k}\right\rangle .
$$

Note that $Q_{i k}$ has a canonical non-zero rational section $q_{i k}$. We have a canonical symmetry isomorphism

$$
Q_{i k} \cong Q_{k i}
$$

sending $q_{i k}$ to $q_{k i}$. If $S$ is the spectrum of a discrete valuation ring we have the following functoriality of $\left(Q_{i k}, q_{i k}\right)$ in passing from $\pi: X \rightarrow S$ to a minimal desingularisation $\rho: X^{\prime} \rightarrow X$ of $X$ over $S$ : the sections $W_{i}, W_{k}$ lift to $\sigma$-invariant sections of $X^{\prime}$, and one obtains the relative dualising sheaf of $X^{\prime}$ over $S$ as the pullback of $\omega$ along $\rho$. It follows that the $V_{i}, V_{k}$ of $X^{\prime}$ over $S$ are obtained by pullback as well, and so the formation of $Q_{i k}$ and its canonical rational section $q_{i k}$ are compatible with the passage from $X$ to $X^{\prime}$.

Assume that $S$ is an integral scheme.

Proposition 2.1. There exists a canonical isomorphism:

$$
\varphi_{i k}:\langle\omega, \omega\rangle \stackrel{\cong}{\rightrightarrows} Q_{i k}
$$

of line bundles on $S$, compatible with any dominant base change. Let $K$ be either a complete discrete valuation field or $\mathbb{R}$ or $\mathbb{C}$ and let $\bar{K}$ be an algebraic closure of $K$. If $S=\operatorname{Spec}(\bar{K})$, then $\varphi_{i k}$ is an isometry for the admissible metrics on both $\langle\omega, \omega\rangle$ and $Q_{i k}$.

Proof. By construction of $V_{i}$ we have a canonical isomorphism:

$$
\omega \stackrel{\cong}{\rightarrow}(2 g-2) W_{i}+V_{i}-(2 g-1) \pi^{*}\left\langle W_{i}, W_{i}\right\rangle
$$

on $X$. Hence we have:

$$
(2 g-2)\left(W_{i}-W_{k}\right) \stackrel{\cong}{\rightrightarrows} V_{k}-V_{i}+(2 g-1) \pi^{*}\left\langle W_{i}, W_{i}\right\rangle-(2 g-1) \pi^{*}\left\langle W_{k}, W_{k}\right\rangle,
$$


canonically. Using pullback along $W_{i}$ and $W_{k}$ we find canonical isomorphisms:

$$
\begin{aligned}
(2 g-2)\left\langle W_{i}-W_{k}, W_{i}-W_{k}\right\rangle & \stackrel{\cong}{\rightrightarrows}\left\langle V_{k}-V_{i}, W_{i}-W_{k}\right\rangle \\
& \cong\left\langle W_{i}, V_{k}\right\rangle+\left\langle V_{i}, W_{k}\right\rangle .
\end{aligned}
$$

Hence we find:

$$
(2 g-2)\left(\left\langle W_{i}, W_{i}\right\rangle+\left\langle W_{k}, W_{k}\right\rangle\right) \stackrel{\cong}{\rightrightarrows}\left\langle W_{i}, V_{k}\right\rangle+\left\langle V_{i}, W_{k}\right\rangle+4(g-1)\left\langle W_{i}, W_{k}\right\rangle .
$$

Also from (2.2) one obtains:

$$
-(2 g-1)\left\langle W_{k}, \pi^{*}\left\langle W_{i}, W_{i}\right\rangle\right) \stackrel{\cong}{\rightrightarrows}-\left\langle W_{k}, W_{k}\right\rangle-(2 g-2)\left\langle W_{i}, W_{k}\right\rangle-\left\langle V_{i}, W_{k}\right\rangle
$$

by using the adjunction isomorphism:

$$
\left\langle W_{k}, \omega\right\rangle \stackrel{\cong}{\longrightarrow}-\left\langle W_{k}, W_{k}\right\rangle
$$

and likewise:

$$
-(2 g-1)\left\langle W_{i}, \pi^{*}\left\langle W_{k}, W_{k}\right\rangle\right) \stackrel{\cong}{\longrightarrow}-\left\langle W_{i}, W_{i}\right\rangle-(2 g-2)\left\langle W_{i}, W_{k}\right\rangle-\left\langle W_{i}, V_{k}\right\rangle .
$$

Adding these two isomorphisms, multiplying by $2 g-2$, and using (2.4) we obtain:

$$
\begin{gathered}
-(2 g-2)(2 g-1)\left(\left\langle W_{i}, \pi^{*}\left\langle W_{k}, W_{k}\right\rangle\right\rangle+\left\langle W_{k}, \pi^{*}\left\langle W_{i}, W_{i}\right\rangle\right\rangle\right) \stackrel{\cong}{\rightarrow} \\
-(2 g-1)\left(\left\langle W_{i}, V_{k}\right\rangle+\left\langle V_{i}, W_{k}\right\rangle\right)-4(g-1)(2 g-1)\left\langle W_{i}, W_{k}\right\rangle .
\end{gathered}
$$

Now note that:

$$
\begin{aligned}
\langle\omega, \omega\rangle & \cong \\
& -(2 g-2)(2 g-1)\left(\left\langle W_{i}, \pi^{*}\left\langle W_{k}, W_{k}\right\rangle\right\rangle+\left\langle W_{k}, \pi^{*}\left\langle W_{i}, W_{i}\right\rangle\right\rangle\right) .
\end{aligned}
$$

Plugging in the previous result gives the required isomorphism.

To see that $\varphi_{i k}$ is an isometry for the admissible metrics on both sides if $S=$ $\operatorname{Spec}(\bar{K})$ with $K$ a complete discretely valued field or $K=\mathbb{R}$ or $\mathbb{C}$ it suffices to verify that both (2.2) and the adjunction isomorphism (2.5) are isometries for the admissible metrics. But that the adjunction isomorphism (2.5) is an isometry for the admissible metrics is precisely in [17, sections 2.7 (Archimedean case) and 4.1 (non-Archimedean case). That (2.2) is an isometry for the admissible metrics on both sides can be seen as follows. The curvature form (see [17, section 2.5) of both the left and right hand side of (2.2) is equal to $(2 g-2) \mu$, where $\mu$ is the admissible metric on the reduction graph of $X$ (if $K$ is a complete discretely valued field) or the Arakelov $(1,1)$-form on $X(\bar{K})$ (if $K=\mathbb{R}$ or $\mathbb{C}$ ). This implies (see once again [17, section 2.5) that the quotient of the admissible metric on $\omega$ and the metric put on $\omega$ via the isomorphism (2.2) is constant on $X \otimes \bar{K}$. By restricting the isomorphism (2.2) to $W_{i}$ on $X \otimes \bar{K}$ we find the adjunction isomorphism $\left\langle W_{i}, \omega\right\rangle \stackrel{\cong}{\rightarrow}-\left\langle W_{i}, W_{i}\right\rangle$. As this is an isometry, so is (2.2).

\section{MAIN RESUlT}

In this section we prove our main result and derive Theorem $\mathrm{C}$ from it. Let $S$ be an integral scheme and let $\pi: X \rightarrow S$ be a generically smooth semistable hyperelliptic curve of genus $g \geq 2$ over $S$.

Assume that a triple $\left(W_{i}, W_{j}, W_{k}\right)$ of $\sigma$-invariant sections of $\pi$ is given with image in the smooth locus of $\pi$. Assume as well that the generic characteristic of $S$ is not equal to 2 . We view the element $\ell_{i j k}^{2 g}$, defined fiber by fiber along the non-empty open subscheme of $S$ where $\pi$ is smooth and the residue characteristic 
is not 2, as a rational section of the structure sheaf $\mathcal{O}_{S}$ of $S$. On the other hand, from Proposition 2.1 we obtain a canonical isomorphism:

$$
\psi_{i j k}=\varphi_{i k} \otimes \varphi_{j k}^{-1}:-Q_{i k}+Q_{j k} \stackrel{\cong}{\longrightarrow} \mathcal{O}_{S},
$$

compatible with dominant base change, and isometric for the admissible metrics on both sides. This yields a rational section of $\mathcal{O}_{S}$ by taking the image of $q_{i k}^{-1} \otimes q_{j k}$ under $\psi_{i j k}$. Our main result is that the image of $q_{i k}^{-1} \otimes q_{j k}$ under $\psi_{i j k}$ is essentially a power of $\ell_{i j k}^{2 g}$.

Theorem 3.1. $\psi_{i j k}$ maps the rational section $q_{i k}^{-1} \otimes q_{j k}$ of $-Q_{i k}+Q_{j k}$ to the rational section $\left(-\ell_{i j k}^{2 g}\right)^{g-1}$ of $\mathcal{O}_{S}$.

A first step in the proof is the following result.

Proposition 3.2. Assume that $S$ is the spectrum of a discrete valuation ring $R$, that $X$ is regular, and that $\pi$ is smooth if the residue characteristic of $R$ is equal to 2. Then the formula:

$$
-\nu\left(q_{i k}\right)+\nu\left(q_{j k}\right)=2 g(g-1) \nu\left(\ell_{i j k}\right)
$$

holds, where $\nu(\cdot)$ denotes order of vanishing along the closed point of $S$.

Proof. We recall that:

$$
Q_{i k}=-4 g(g-1)\left\langle W_{i}, W_{k}\right\rangle-\left\langle W_{i}, V_{k}\right\rangle-\left\langle V_{i}, W_{k}\right\rangle+\left\langle V_{i}, V_{k}\right\rangle .
$$

As $\left\langle(2 g-2) W_{i}-\omega+V_{i}, V_{k}\right\rangle$ is canonically trivial we have a canonical isomorphism:

$$
Q_{i k} \cong-4 g(g-1)\left\langle W_{i}, W_{k}\right\rangle-(2 g-1)\left\langle W_{i}, V_{k}\right\rangle-\left\langle V_{i}, W_{k}\right\rangle+\left\langle V_{k}, \omega\right\rangle
$$

and hence:

$-\nu\left(q_{i k}\right)+\nu\left(q_{j k}\right)=4 g(g-1)\left(W_{i}-W_{j}, W_{k}\right)+(2 g-1)\left(W_{i}-W_{j}, V_{k}\right)+\left(V_{i}-V_{j}, W_{k}\right)$, where $(\cdot, \cdot)$ denotes intersection product on $\operatorname{Div}(X)$. Our task is thus to show that: $4 g(g-1)\left(W_{i}-W_{j}, W_{k}\right)+(2 g-1)\left(W_{i}-W_{j}, V_{k}\right)+\left(V_{i}-V_{j}, W_{k}\right)=2 g(g-1) \nu\left(\ell_{i j k}\right)$. Let $S^{\prime} \rightarrow S$ be any finite cover of $S$, and let $X^{\prime} \rightarrow S^{\prime}$ be the minimal desingularisation of the base change of $X \rightarrow S$ along $S^{\prime} \rightarrow S$. By functoriality of $\left(Q_{i k}, q_{i k}\right)$ and invariance under pullback it suffices to prove the formula for $X^{\prime} \rightarrow S^{\prime}$. We start with the case that the residue characteristic of $R$ is not equal to 2 . Let $m$ be the maximal ideal of $R$ and let $K$ be the fraction field of $R$. By [7, Lemma 4.1] we may assume that $X \otimes K$ has an affine equation $y^{2}=\prod_{r=1}^{2 g+2}\left(x-a_{r}\right)$ such that:

- the $a_{r}$ are distinct elements of $R$;

- the valuations $\nu\left(a_{r}-a_{s}\right)$ are even for $r \neq s$;

- the $a_{r}$ lie in at least 3 distinct residue classes of $R$ modulo $m$.

As the sections $W_{i}, W_{j}, W_{k}$ are disjoint we are reduced to showing that:

$$
(2 g-1)\left(W_{i}-W_{j}, V_{k}\right)+\left(V_{i}-V_{j}, W_{k}\right)=2 g(g-1) \nu\left(\ell_{i j k}\right) .
$$

Let $\alpha$ be the subset $\left\{a_{1}, \ldots, a_{2 g+2}\right\}$ of $R$. To $\alpha$ we associate a finite tree $T$, as follows: for each positive integer $n$ let $\rho_{n}: \alpha \rightarrow R / m^{n}$ be the canonical residue map. Define $\Lambda_{n}$ to be the set of residue classes $\lambda$ in $R / m^{n}$ such that $\rho_{n}^{-1}(\lambda) \subset \alpha$ has at least 2 elements. The vertices of $T$ are then the elements $\lambda$ of $\Lambda_{n}$ for $n$ running through the non-negative integers; there are only finitely many such $\lambda$. The edges of $T$ are the pairs $\left(\lambda, \lambda^{\prime}\right)$ of vertices $\lambda, \lambda^{\prime}$ where $\lambda \in \Lambda_{n}, \lambda^{\prime} \in \Lambda_{n+1}$ and 
$\lambda^{\prime} \mapsto \lambda$ under the natural map $\Lambda_{n+1} \rightarrow \Lambda_{n}$, for some $n$. If $\lambda$ is a vertex of $T$ there is a unique $n$ such that $\lambda \in \Lambda_{n}$; we call $n$ the level of $\lambda$.

Let $F$ be the special fiber of $\pi$ and let $\Gamma$ be the dual graph of $F$. According to [1, Section 5] or [7, Section 4] there is a natural graph morphism $\varphi: \Gamma \rightarrow T$. If $C$ is a vertex of $\Gamma$ we denote by $\lambda_{C}$ the image of $C$ in $T$, and by $n_{C}$ the level of $\lambda_{C}$. For each $r=1, \ldots, 2 g+2$ we denote by $C_{r}$ the unique irreducible component of $F$ through which the $\sigma$-invariant section of $\pi$ corresponding to $a_{r}$ passes, by $\lambda_{r}$ the image of $C_{r}$ in $T$, and by $n_{r}$ the level of $\lambda_{r}$. By construction of $\varphi$ the element $a_{r}$ is a representative of the class $\lambda_{r}$ and $n_{r}=\max _{s \neq r} \nu\left(a_{r}-a_{s}\right)$.

For each irreducible component $C$ of $F$ we choose a representative $a_{C}$ of $\lambda_{C}$ in $\alpha$. If $f$ is a non-zero rational function on $X$ we denote by $\nu_{C}(f)$ the multiplicity of $f$ along $C$. We have:

$$
\nu_{C}\left(x-a_{r}\right)=\min \left\{n_{C}, \nu\left(a_{C}-a_{r}\right)\right\},
$$

independent of the choice of $a_{C}$, by [7, proof of Lemma 5.1. In particular:

$$
\nu_{C_{k}}\left(x-a_{r}\right)= \begin{cases}\nu\left(a_{k}-a_{r}\right) & r \neq k \\ n_{k} & r=k\end{cases}
$$

and so:

$$
\nu_{C_{k}}(y)=\frac{1}{2} n_{k}+\frac{1}{2} \sum_{r \neq k} \nu\left(a_{k}-a_{r}\right) .
$$

Now write $V_{k}=\sum_{C} \mu_{k}(C) \cdot C$ with $\mu_{k}(C) \in \mathbb{Z}$ and with $C$ running through the irreducible components of $F$. We claim that:

$\mu_{k}(C)=(g-1) \min \left\{n_{C}, \nu\left(a_{k}-a_{C}\right)\right\}-\nu_{C}(y)+n_{C}-\left(g-\frac{1}{2}\right) n_{k}+\frac{1}{2} \sum_{r \neq k} \nu\left(a_{k}-a_{r}\right)$

for all $C$. To see this, consider the rational section:

$$
\omega_{k}=\left(x-a_{k}\right)^{g-1} \frac{\mathrm{d} x}{y}
$$

of $\omega$. Let $\mu_{k}^{\prime}(C)=(g-1) \nu_{C}\left(x-a_{k}\right)-\nu_{C}(y)+n_{C}$. According to [7, Lemma 5.2] we have:

$$
\operatorname{div}_{X} \omega_{k}=(2 g-2) W_{k}+\sum_{C} \mu_{k}^{\prime}(C) \cdot C .
$$

It follows that $V_{k}$ and $\sum_{C} \mu_{k}^{\prime}(C) \cdot C$ differ by a multiple of $F$. As $W_{k}^{*} V_{k}$ is trivial we find that:

$$
V_{k}=\sum_{C}\left(\mu_{k}^{\prime}(C)-\mu_{k}^{\prime}\left(C_{k}\right)\right) \cdot C
$$

As we have:

$$
\begin{aligned}
\mu_{k}^{\prime}\left(C_{k}\right) & =(g-1) \nu_{C_{k}}\left(x-a_{k}\right)-\nu_{C_{k}}(y)+n_{k} \\
& =(g-1) n_{k}-\frac{1}{2} n_{k}-\frac{1}{2} \sum_{r \neq k} \nu\left(a_{r}-a_{k}\right)+n_{k} \\
& =\left(g-\frac{1}{2}\right) n_{k}-\frac{1}{2} \sum_{r \neq k} \nu\left(a_{r}-a_{k}\right),
\end{aligned}
$$


the claim follows. As an immediate consequence we have:

$$
\begin{aligned}
\left(W_{i}, V_{k}\right)= & \mu_{k}\left(C_{i}\right) \\
= & (g-1) \min \left\{n_{i}, \nu\left(a_{k}-a_{i}\right)\right\}-\nu_{C_{i}}(y)+n_{i} \\
& \quad-\left(g-\frac{1}{2}\right) n_{k}+\frac{1}{2} \sum_{r \neq k} \nu\left(a_{k}-a_{r}\right) \\
= & (g-1) \nu\left(a_{k}-a_{i}\right)+\frac{1}{2} n_{i}-\frac{1}{2} \sum_{r \neq i} \nu\left(a_{r}-a_{i}\right) \\
& \quad-\left(g-\frac{1}{2}\right) n_{k}+\frac{1}{2} \sum_{r \neq k} \nu\left(a_{k}-a_{r}\right),
\end{aligned}
$$

so that:

$$
\left(W_{i}-W_{j}, V_{k}\right)=(g-1) \nu\left(\frac{a_{i}-a_{k}}{a_{j}-a_{k}}\right)+\frac{1}{2}\left(n_{i}-n_{j}\right)+\frac{1}{2} \sum_{r \neq i, j} \nu\left(\frac{a_{j}-a_{r}}{a_{i}-a_{r}}\right),
$$

and in a similar fashion:

$$
\left(V_{i}-V_{j}, W_{k}\right)=(g-1) \nu\left(\frac{a_{i}-a_{k}}{a_{j}-a_{k}}\right)-\left(g-\frac{1}{2}\right)\left(n_{i}-n_{j}\right)-\frac{1}{2} \sum_{r \neq i, j} \nu\left(\frac{a_{j}-a_{r}}{a_{i}-a_{r}}\right) .
$$

This leads to:

$$
\begin{aligned}
(2 g-1)\left(W_{i}-W_{j}, V_{k}\right)+\left(V_{i}-V_{j}, W_{k}\right)= & 2 g(g-1) \nu\left(\frac{a_{i}-a_{k}}{a_{j}-a_{k}}\right) \\
& +(g-1) \sum_{r \neq i, j} \nu\left(\frac{a_{j}-a_{r}}{a_{i}-a_{r}}\right),
\end{aligned}
$$

and the required formula follows.

Next we consider the case that $R$ does have residue characteristic equal to 2 . We have that $X \rightarrow S$ is smooth, and we may assume that all Weierstrass points of $X \otimes K$ are rational over $K$, hence extend to sections of $\pi$. The divisors $V_{i}, V_{j}$ and $V_{k}$ are empty, and we are reduced to showing that simply:

$$
2\left(W_{i}-W_{j}, W_{k}\right)=\nu\left(\ell_{i j k}\right) .
$$

Let $h: X \rightarrow Y$ be the quotient of $X$ by $\sigma$. According to [10, Section 5] we have that $Y \rightarrow S$ is a smooth proper family of curves of genus 0 , and $h$ is finite flat of degree 2. Let $P_{i}, P_{j}, P_{k}: S \rightarrow Y$ denote the sections $W_{i}, W_{j}, W_{k}$, composed with $h$. By the projection formula we have:

$$
2\left(W_{i}, W_{k}\right)=\left(P_{i}, P_{k}\right), \quad 2\left(W_{j}, W_{k}\right)=\left(P_{j}, P_{k}\right) .
$$

By [7, Lemma 6.1] we may assume that on an affine open subset $X$ is given by an equation $y^{2}+p(x) y=q(x)$ with $p, q \in R[x]$ such that $p^{2}+4 q$ is a separable polynomial of degree $d=2 g+2$. As $p=0$ defines the fixed point subscheme of the hyperelliptic involution on the special fiber of $X \rightarrow S$ the coefficients of $p$ generate the unit ideal in $R$. It follows that we may even assume after a translation that $p(0)$ is a unit in $R$ and subsequently after making a coordinate transformation $x \mapsto x^{-1}$ that $p$ has degree $g+1$ and leading coefficient a unit in $R$. Write $f=p^{2}+4 q \in R[x]$. Then $f$ has leading coefficient a unit in $R$ as well. We can write $f=b \cdot \prod_{r=1}^{2 g+2}\left(x-a_{r}\right)$ in $K[x]$; then $b \in R^{\times}$, and by Gauss's Lemma the $a_{i}$ are actually in $R$. Let 
$a_{i}, a_{j}, a_{k} \in R$ correspond to $P_{i}, P_{j}, P_{k}$. As $y^{2}=f(x)$ is a hyperelliptic equation for $X \otimes K$ we have:

$$
\ell_{i j k}=\frac{a_{i}-a_{k}}{a_{j}-a_{k}} \sqrt[2 g]{\prod_{r \neq i, j} \frac{a_{j}-a_{r}}{a_{i}-a_{r}}}=\frac{a_{i}-a_{k}}{a_{j}-a_{k}} \sqrt[2 g]{-\frac{f^{\prime}\left(a_{j}\right)}{f^{\prime}\left(a_{i}\right)}}
$$

in $K^{\times} / \mu_{2 g}$. Since by the projection formula:

$$
2\left(W_{i}, W_{k}\right)=\nu\left(a_{i}-a_{k}\right), \quad 2\left(W_{j}, W_{k}\right)=\nu\left(a_{j}-a_{k}\right),
$$

we are done once we prove that $f^{\prime}\left(a_{j}\right) / f^{\prime}\left(a_{i}\right)$ is a unit in $R$. Let $a_{r}$ be an arbitrary root of $f$; we will show that $\nu\left(f^{\prime}\left(a_{r}\right)\right)=\nu(4)$, so that $\nu\left(f^{\prime}\left(a_{r}\right)\right)$ is independent of $r$. From the equation $p\left(a_{r}\right)^{2}+4 q\left(a_{r}\right)=0$ we obtain first of all that $p\left(a_{r}\right)$ is divisible by 2 in $R$. From $f=p^{2}+4 q$ in $R[x]$ we obtain $f^{\prime}\left(a_{r}\right)=2 p\left(a_{r}\right) p^{\prime}\left(a_{r}\right)+4 q^{\prime}\left(a_{r}\right)$ so that 4 divides $f^{\prime}\left(a_{r}\right)$ in $R$ and hence $\nu\left(f^{\prime}\left(a_{r}\right)\right) \geq \nu(4)$. According to [7, Proposition 6.3] we have however:

$$
\sum_{s=1}^{2 g+2} \nu\left(f^{\prime}\left(a_{s}\right)\right)=\nu\left(b^{d} \cdot \prod_{s \neq t}\left(a_{s}-a_{t}\right)\right)=\nu\left(b^{2 d-2} \cdot \prod_{s \neq t}\left(a_{s}-a_{t}\right)\right)=(2 g+2) \nu(4),
$$

and we conclude that $\nu\left(f^{\prime}\left(a_{r}\right)\right)=\nu(4)$ as required.

Let $\mathcal{I}_{g, 3}^{*}$ be the moduli stack, over $\mathbb{Z}[1 / 2]$, of pairs $\left(X \rightarrow S,\left(W_{i}, W_{j}, W_{k}\right)\right)$ with $S$ a $\mathbb{Z}[1 / 2]$-scheme, $X \rightarrow S$ a smooth hyperelliptic curve of genus $g$, and $\left(W_{i}, W_{j}, W_{k}\right)$ a triple of distinct $\sigma$-invariant sections of $X \rightarrow S$.

Lemma 3.3. The moduli stack $\mathcal{I}_{g, 3}^{*}$ is irreducible, and smooth over $\operatorname{Spec}(\mathbb{Z}[1 / 2])$.

Proof. Let $\mathcal{I}_{g}$ be the moduli stack of smooth hyperelliptic curves of genus $g$ over $\mathbb{Z}[1 / 2]$, and let $\mathcal{U}_{1}$ be the universal hyperelliptic curve over $\mathcal{I}_{g}$. Define inductively for $n \geq 2$ the algebraic stack $\mathcal{U}_{n}$ over $\mathcal{U}_{n-1}$ as the base change of $\mathcal{U}_{1} \rightarrow \mathcal{I}_{g}$ along $\mathcal{U}_{n-1} \rightarrow \mathcal{I}_{g}$. In particular, the algebraic stack $\mathcal{U}_{n}$ is a smooth hyperelliptic curve over $\mathcal{U}_{n-1}$, for all $n \geq 2$. Let $\mathcal{V}_{n}$ be the fixed point substack of the hyperelliptic involution of $\mathcal{U}_{n}$ over $\mathcal{U}_{n-1}$; then $\mathcal{V}_{n} \rightarrow \mathcal{U}_{n}$ is a closed immersion, and the induced map $\mathcal{V}_{n} \rightarrow \mathcal{U}_{n-1}$ is finite étale by [10, Corollary 6.8]. We are interested in the cases $n=1,2,3$. Put:

$$
\mathcal{A}=\mathcal{V}_{2} \times_{\mathcal{U}_{1}} \mathcal{V}_{1}, \quad \mathcal{B}=\mathcal{V}_{3} \times{ }_{\mathcal{U}_{2}} \mathcal{V}_{2}, \quad \mathcal{C}=\mathcal{A} \times \mathcal{V}_{2} \mathcal{B}
$$

As $\mathcal{U}_{1}$ is naturally the moduli stack of 1 -pointed hyperelliptic curves over $\mathbb{Z}[1 / 2]$, we have a natural map $\mathcal{I}_{g, 3}^{*} \rightarrow \mathcal{U}_{3}$. It factors via the closed immersion $\mathcal{C} \rightarrow \mathcal{U}_{3}$, and the induced map $\mathcal{I}_{g, 3}^{*} \rightarrow \mathcal{C}$ is an open immersion. Since $\mathcal{C} \rightarrow \mathcal{I}_{g}$ is étale and the structure map $\mathcal{I}_{g} \rightarrow \operatorname{Spec}(\mathbb{Z}[1 / 2]$ ) is smooth (see [ 8 , Theorem 3]), we obtain that $\mathcal{I}_{g, 3}^{*}$ is smooth over $\operatorname{Spec}(\mathbb{Z}[1 / 2])$. It follows that the generic points of $\mathcal{I}_{g, 3}^{*}$ all lie above the generic point of $\operatorname{Spec}(\mathbb{Z}[1 / 2])$. But $\mathcal{I}_{g, 3}^{*} \otimes \mathbb{Q}$ is a quotient of the moduli stack of $2 g+2$ distinct points on $\mathbb{P}^{1}$, which is irreducible, hence $\mathcal{I}_{g, 3}^{*} \otimes \mathbb{Q}$ is irreducible. It follows that $\mathcal{I}_{g, 3}^{*}$ is irreducible as well.

We need a suitable compactification of $\mathcal{I}_{g, 3}^{*}$. Note that we have a natural closed immersion $\mathcal{I}_{g, 3}^{*} \rightarrow \mathcal{M}_{g, 3}$ where $\mathcal{M}_{g, 3}$ is the moduli stack, over $\mathbb{Z}[1 / 2]$, of 3-pointed smooth projective curves of genus $g$. We let $\overline{\mathcal{I}}_{g, 3}^{*}$ be the stack-theoretic closure of $\mathcal{I}_{g, 3}^{*}$ in $\overline{\mathcal{M}}_{g, 3}$, the Knudsen-Mumford compactification of $\mathcal{M}_{g, 3}$. Then $\overline{\mathcal{I}}_{g, 3}^{*}$ is integral, and proper and flat over $\mathbb{Z}[1 / 2]$. We have $\ell_{i j k}^{2 g}$ and, via $\psi_{i j k}$, also $q_{i k}^{-1} \otimes q_{j k}$ 
as rational sections of the structure sheaf of $\overline{\mathcal{I}}_{g, 3}^{*}$. In fact, for every generically smooth semistable hyperelliptic curve $\pi: X \rightarrow S$ with $S$ an integral $\mathbb{Z}[1 / 2]$-scheme together with a triple of distinct $\sigma$-invariant sections with image in the smooth locus of $\pi$, we have a natural period map $S \rightarrow \overline{\mathcal{I}}_{g, 3}^{*}$ such that both $q_{i k}^{-1} \otimes q_{j k}$ and $\ell_{i j k}^{2 g}$ associated to $\pi$ on $S$ are obtained by pullback from $\overline{\mathcal{I}}_{g, 3}^{*}$.

It suffices therefore to prove Theorem 3.1 for the tautological curve over $\overline{\mathcal{I}}_{g, 3}^{*}$.

Proof of Theorem 3.1. We first prove that $q_{i k}^{-1} \otimes q_{j k}$ and $\ell_{i j k}^{2 g(g-1)}$ on $\overline{\mathcal{I}}_{g, 3}^{*}$ differ by a unit in $\mathbb{Z}[1 / 2]$. Let $\nu:{ }^{\prime} \overline{\mathcal{I}}_{g, 3}^{*} \rightarrow \overline{\mathcal{I}}_{g, 3}^{*}$ be the normalisation of $\overline{\mathcal{I}}_{g, 3}^{*}$. As $\mathbb{Z}[1 / 2]$ is an excellent ring, the morphism $\nu$ is finite birational. In particular ${ }^{\prime} \overline{\mathcal{I}}_{g, 3}^{*}$ and $\overline{\mathcal{I}}_{g, 3}^{*}$ are isomorphic over an open dense substack. Hence, in order to prove that $q_{i k}^{-1} \otimes q_{j k}$ and $\ell_{i j k}^{2 g(g-1)}$ differ by a unit in $\mathbb{Z}[1 / 2]$ it suffices to prove that their pullbacks along $\nu$ do so over ${ }^{\prime} \overline{\mathcal{I}}_{g, 3}^{*}$. This goes in two steps: first we prove that $q_{i k}^{-1} \otimes q_{j k}$ and $\ell_{i j k}^{2 g(g-1)}$ differ by an invertible regular function on ${ }^{\prime} \overline{\mathcal{I}}_{g, 3}^{*}$, and then that the set of such functions is precisely $\mathbb{Z}[1 / 2]^{\times}$. As to the first step, since ${ }^{\prime} \overline{\mathcal{I}}_{g, 3}^{*}$ is normal, it suffices to prove that for every point $x^{\prime}$ of ${ }^{\prime} \overline{\mathcal{I}}_{g, 3}^{*}$ of height 1 , the sections $q_{i k}^{-1} \otimes q_{j k}$ and $\ell_{i j k}^{2 g(g-1)}$ differ by a unit in the local $\operatorname{ring} \mathcal{O}_{x^{\prime}}$ at $x^{\prime}$. So let $x^{\prime}$ be a point of height 1 on ${ }^{\prime} \overline{\mathcal{I}}_{g, 3}^{*}$. Let $\operatorname{Spec}\left(\mathcal{O}_{x^{\prime}}\right) \rightarrow{ }^{\prime} \overline{\mathcal{I}}_{g, 3}^{*} \stackrel{\nu}{\rightarrow} \overline{\mathcal{I}}_{g, 3}^{*}$ be the canonical map. Then the generic point of $\operatorname{Spec}\left(\mathcal{O}_{x^{\prime}}\right)$ maps to the generic point of $\overline{\mathcal{I}}_{g, 3}^{*}$ and by pullback we obtain a generically smooth stable hyperelliptic curve $\pi^{\prime}: X^{\prime} \rightarrow \operatorname{Spec}\left(\mathcal{O}_{x^{\prime}}\right)$ over $\mathcal{O}_{x^{\prime}}$ together with a triple of distinct $\sigma$-invariant sections with image in the smooth locus of $\pi^{\prime}$. By functoriality of $\left(Q_{i k}, q_{i k}\right)$ in passing from $X^{\prime}$ to a minimal desingularisation of $X^{\prime}$ over $\operatorname{Spec}\left(\mathcal{O}_{x^{\prime}}\right)$ we may assume for our purposes that $X^{\prime}$ is itself regular and semistable over $\operatorname{Spec}\left(\mathcal{O}_{x^{\prime}}\right)$. Proposition 3.2 then gives us precisely what we need.

Next let $p:{ }^{\prime} \overline{\mathcal{I}}_{g, 3}^{*} \rightarrow \operatorname{Spec}(\mathbb{Z}[1 / 2])$ be the structure map; we claim that $p_{*} \mathcal{O}_{, \overline{\mathcal{I}}_{g, 3}^{*}}$ is equal to $\mathcal{O}_{\mathrm{Spec}(\mathbb{Z}[1 / 2])}$. This will prove that the set of invertible regular functions on ${ }^{\prime} \overline{\mathcal{I}}_{g, 3}^{*}$ is equal to $\mathbb{Z}[1 / 2]^{\times}$. The claim follows from: (i) $p$ is proper, and (ii) $p$ is flat and ' $\overline{\mathcal{I}}_{g, 3}^{*} \otimes \mathbb{Q}$ is irreducible. Indeed, if $p$ is proper and flat, then $p_{*} \mathcal{O}_{,} \overline{\mathcal{I}}_{g, 3}^{*}$ is a finite torsion-free $\mathcal{O}_{\operatorname{Spec}(\mathbb{Z}[1 / 2])}$-module. If moreover ${ }^{\prime} \overline{\mathcal{I}}_{g, 3}^{*} \otimes \mathbb{Q}$ is irreducible, then $p_{*} \mathcal{O}_{\overline{\mathcal{I}}_{g, 3}^{*}}$ has generic rank 1 , and $p_{*} \mathcal{O}_{\overline{\mathcal{I}}_{g, 3}^{*}}=\mathcal{O}_{\mathrm{Spec}(\mathbb{Z}[1 / 2])}$ follows. That (i) is satisfied is clear as both $\nu$ and $\overline{\mathcal{I}}_{g, 3}^{*} \rightarrow \operatorname{Spec}(\mathbb{Z}[1 / 2])$ are proper. To see (ii), recall that $\overline{\mathcal{I}}_{g, 3}^{*}$ is irreducible and has its generic point mapping to the generic point of $\operatorname{Spec}(\mathbb{Z}[1 / 2])$. The same holds for ' $\overline{\mathcal{I}}_{g, 3}^{*}$ since $\nu$ is birational; in particular ${ }^{\prime} \overline{\mathcal{I}}_{g, 3}^{*}$ is flat over $\mathbb{Z}[1 / 2]$.

The next step is to prove that the unit $u$ in $\mathbb{Z}[1 / 2]$ connecting $q_{i k}^{-1} \otimes q_{j k}$ and $\ell_{i j k}^{2 g(g-1)}$ is either +1 or -1 . For this we use a smooth hyperelliptic curve $X \rightarrow S$ with a triple of $\sigma$-invariant sections over the spectrum $S$ of a discrete valuation ring with residue characteristic equal to 2 , and generic characteristic zero (such data exist). We have a period map $S \otimes \mathbb{Q} \rightarrow \overline{\mathcal{I}}_{g, 3}^{*} \otimes \mathbb{Q}$ and applying once more Proposition 3.2 we see that the exponent of 2 in $u$ is vanishing.

We finish by proving that $u=(-1)^{g-1}$. Cyclic permutation of the three $\sigma$ invariant sections in the moduli data induces a group of automorphisms of the moduli stack $\mathcal{I}_{g, 3}^{*}$ of order three. Its action on the regular functions on $\mathcal{I}_{g, 3}^{*}$ yields the regular functions $\ell_{j k i}^{2 g}$ and $\ell_{k i j}^{2 g}$ from $\ell_{i j k}^{2 g}$, as well as the functions $q_{j i}^{-1} \otimes q_{k i}$ and 
$q_{k j}^{-1} \otimes q_{i j}$ from $q_{i k}^{-1} \otimes q_{j k}$. A small computation shows the cocyle relation:

$$
\ell_{i j k}^{2 g} \cdot \ell_{j k i}^{2 g} \cdot \ell_{k i j}^{2 g}=-1,
$$

whereas we have:

$$
q_{i k}^{-1} \otimes q_{j k} \otimes q_{j i}^{-1} \otimes q_{k i} \otimes q_{k j}^{-1} \otimes q_{i j}=1
$$

by the canonical symmetry isomorphism (2.1). Now write $\ell_{i j k}^{2 g(g-1)}=u \cdot q_{i k}^{-1} \otimes q_{j k}$. As $u$, being a constant function, is left invariant by any automorphism of $\mathcal{I}_{g, 3}^{*}$, we obtain the identities:

$$
\ell_{j k i}^{2 g(g-1)}=u \cdot q_{j i}^{-1} \otimes q_{k i}, \quad \ell_{k i j}^{2 g(g-1)}=u \cdot q_{k j}^{-1} \otimes q_{i j} .
$$

Combining the cocycle relations with these identities yields $u=u^{3}=(-1)^{g-1}$. The proof of Theorem 3.1 is thereby complete.

We are now ready to prove Theorem C. Let $K$ be a field which is either a complete discrete valuation field, or $\mathbb{R}$ or $\mathbb{C}$. Let $\bar{K}$ be an algebraic closure of $K$ and endow $\bar{K}$ with its canonical absolute value $|\cdot|$. This absolute value is defined as follows: if $K$ is a complete discrete valuation field, endow $K$ with the absolute value $|\cdot|_{K}$ such that $|\pi|=1 / e$ for a uniformizer $\pi$ of $K$; we get an absolute value $|\cdot|$ on $\bar{K}$ by taking the unique extension of $|\cdot|_{K}$ to $\bar{K}$. If $K=\mathbb{R}$ or $K=\mathbb{C}$ we endow $\bar{K}=\mathbb{C}$ with the standard Euclidean norm.

Assume that $K$ does not have characteristic 2 .

Theorem 3.4. Let $X$ be a hyperelliptic curve over $K$, and let $(,)_{a}$ be Zhang's admissible pairing on $\operatorname{Div}(X \otimes \bar{K})$. Let $w_{i}, w_{j}, w_{k}$ be three distinct Weierstrass points on $X \otimes \bar{K}$. Then the formula:

$$
\left(w_{i}-w_{j}, w_{k}\right)_{a}=-\frac{1}{2} \log \left|\ell_{i j k}\right|
$$

holds.

Proof. We apply Theorem 3.1 to the morphism $X \otimes \bar{K} \rightarrow \operatorname{Spec}(\bar{K})$. Let $g$ be the genus of $X$. Under the isomorphism $\psi_{i j k}$ the sections $q_{i k}^{-1} \otimes q_{j k}$ and $\left(-\ell_{i j k}^{2 g}\right)^{g-1}$ are identified, and by Proposition 2.1 the isomorphism $\psi_{i j k}$ is an admissible isometry. Let $|\cdot|_{a}$ be the admissible norm on $-Q_{i k}+Q_{j k}$ on $\operatorname{Spec}(\bar{K})$. We obtain:

$$
\begin{aligned}
2 g(g-1) \log \left|\ell_{i j k}\right| & =\log \left|q_{i k}^{-1} \otimes q_{j k}\right|_{a} \\
& =-4 g(g-1)\left(w_{i}-w_{j}, w_{k}\right)_{a},
\end{aligned}
$$

and the theorem follows.

Corollary 3.5. Let $w_{i}, w_{j}, w_{k}, w_{r}$ be four distinct Weierstrass points on $X \otimes \bar{K}$. Let $\mu_{i j k r}$ in $\bar{K}$ be the cross-ratio on $w_{i}, w_{j}, w_{k}, w_{r}$. Then the formula:

$$
\left(w_{i}-w_{j}, w_{k}-w_{r}\right)_{a}=-\frac{1}{2} \log \left|\mu_{i j k r}\right|
$$

holds.

Proof. This follows directly from the identity:

$$
\frac{\ell_{i j k}}{\ell_{i j r}}=\frac{a_{i}-a_{k}}{a_{j}-a_{k}} \cdot \frac{a_{j}-a_{r}}{a_{i}-a_{r}}=\mu_{i j k r}
$$

which is easily checked. 
Remark 3.6. In the case that $K=\mathbb{R}$ or $\mathbb{C}$ the admissible pairing is given by the Arakelov-Green's function $G$ of the compact Riemann surface $X(\bar{K})$. Theorem 3.4 translates into the remarkable formula:

$$
\frac{G\left(w_{i}, w_{k}\right)}{G\left(w_{j}, w_{k}\right)}=\sqrt{\left|\ell_{i j k}\right|} .
$$

It would be interesting to see if one could give a direct proof of this formula that does not use moduli spaces.

\section{THE INVARIANT $\chi$}

In this section we introduce the $\chi$-invariant as announced in the Introduction. The definition may seem rather $a d$ hoc at first sight, but in the function field context the invariant already occurs, as mentioned before, in work of A. Moriwaki 13 and K. Yamaki [16]. In the next section we present a more intrinsic approach to $\chi$, using the arithmetic of symmetric roots.

Let $\pi: X \rightarrow S$ be an arbitrary generically smooth semistable hyperelliptic curve of genus $g$ with $S$ an integral scheme. Let $\omega$ be the relative dualising sheaf of $\pi$, and let $\lambda=\operatorname{det} R \pi_{*} \omega$ be the Hodge bundle on $S$. As is explained in [11, Section 2], the line bundle $(8 g+4) \lambda$ has a canonical non-zero rational section $\Lambda_{g}$, satisfying the following properties. We write $\mathcal{I}_{g}$ for the moduli stack of smooth hyperelliptic curves of genus $g$ over $\mathbb{Z}$.

- the formation of $\Lambda_{g}$ is compatible with dominant base change;

- if $S$ is normal, then $\Lambda_{g}$ is global;

- if $\pi$ is smooth, then $\Lambda_{g}$ is global and nowhere vanishing;

- if $S$ is the spectrum of a field $K$ which is not of characteristic 2, and $y^{2}+p(x) y=q(x)$ is an affine equation of $X$ with $p, q \in K[x]$, one has:

$$
\Lambda_{g}=\left(2^{-(4 g+4)} \cdot D\right)^{g} \cdot\left(\frac{\mathrm{d} x}{2 y+p} \wedge \cdots \wedge \frac{x^{g-1} \mathrm{~d} x}{2 y+p}\right)^{\otimes 8 g+4},
$$

where $D$ is the discriminant of the separable polynomial $p^{2}+4 q \in K[x]$;

- let $\overline{\mathcal{I}}_{g}$ be the stack-theoretic closure of $\mathcal{I}_{g}$ in $\overline{\mathcal{M}}_{g}$, the moduli stack of stable curves of genus $g$ over $\mathbb{Z}$. Then on $S$, the rational section $\Lambda_{g}$ can be obtained as the pullback of a rational section $\Lambda_{g}$ of $(8 g+4) \lambda$ on $\overline{\mathcal{I}}_{g}$.

Now assume that $S=\operatorname{Spec}(K)$ where $K$ is either a complete discrete valuation field or $\mathbb{R}$ or $\mathbb{C}$. Note that we put no restrictions, at this stage, on the characteristic of $K$. The section $\Lambda_{g}$ gives rise to a real-valued invariant $d(X)$ associated to $X$, as follows. If $K$ is non-Archimedean, let $\mathcal{X} \rightarrow \operatorname{Spec}(R)$ be the minimal regular model of $X$, where $R$ is the valuation ring of $K$. Then $d(X)$ is the order of vanishing of $\Lambda_{g}$ along the closed point of $S$. If $K$ is Archimedean, the $\mathbb{C}$-vector space $\mathrm{H}^{0}(X(\mathbb{C}), \omega)$ is equipped with a natural Hermitian inner product $(\omega, \eta) \mapsto \frac{i}{2} \int_{X(\mathbb{C})} \omega \bar{\eta}$, and $d(X)$ is the $-\log$ of the norm of $\Lambda_{g}$ with respect to this inner product.

One can give explicit formulas for $d(X)$. Start again with the case that $K$ is a complete discrete valuation field. Assume that $X$ has semistable reduction over $K$, and as above let $\mathcal{X} \rightarrow \operatorname{Spec}(R)$ be the minimal regular model of $X$. Let $x$ be a singular point in the special fiber of $\mathcal{X}$. We say that $x$ is of type 0 if the local normalisation of the special fiber at $x$ is connected, and that $x$ is of type $i$, where $1 \leq i \leq\lfloor g / 2\rfloor$, if the local normalisation of the special fiber of $\mathcal{X}$ at $x$ is the disjoint union of two semistable curves of genus $g$ and $g-i$. Let $x$ be a singular point 
of type 0 . Let $\sigma$ be the hyperelliptic involution of $\mathcal{X}$. We have the following two possibilities for $x$ :

- $x$ is fixed by $\sigma$. Then we say $x$ is of subtype 0 .

- $x$ is not fixed by $\sigma$. Then the local normalisation of the special fiber of $\mathcal{X}$ at $\{x, \sigma(x)\}$ consists of two connected components of genus $j$ and $g-j-1$, say, where $1 \leq j \leq\lfloor(g-1) / 2\rfloor$. In this case we say that $x$ is of subtype $j$.

Let $\delta_{i}(X)$ for $i=1, \ldots,\lfloor g / 2\rfloor$ be the number of singular points in the special fiber of $\mathcal{X}$ of type $i$, let $\xi_{0}(X)$ be the number of singular points of subtype 0 , and let $\xi_{j}(X)$ for $j=1, \ldots,\lfloor(g-1) / 2\rfloor$ be the number of pairs of nodes of subtype $j$. Then the following equality holds, proved in increasing order of generality by M. Cornalba and J. Harris, I. Kausz, and K. Yamaki [15:

$$
d(X)=g \xi_{0}(X)+\sum_{j=1}^{\lfloor(g-1) / 2\rfloor} 2(j+1)(g-j) \xi_{j}(X)+\sum_{i=1}^{\lfloor g / 2\rfloor} 4 i(g-i) \delta_{i}(X) .
$$

If $K$ equals $\mathbb{R}$ or $\mathbb{C}$, then $d(X)$ can be related to a product of Thetanullwerte, as explained in [5, Section 8]. Let $\tau$ in the Siegel upper half space be a normalised period matrix for $X(\mathbb{C})$ formed on a canonical symplectic basis of $\mathrm{H}_{1}(X(\mathbb{C}), \mathbb{Z})$. Let $\varphi_{g}$ be the level 2 Siegel modular form from [9, Definition 3.1] and put:

$$
\Delta_{g}=2^{-(4 g+4) n} \varphi_{g}
$$

where $n=\left(\begin{array}{c}2 g \\ g+1\end{array}\right)$. Then the real number $\left\|\Delta_{g}\right\|(X)=(\operatorname{det} \operatorname{Im} \tau)^{2 r}\left|\Delta_{g}(\tau)\right|$ is independent of the choice of $\tau$, where $r=\left(\begin{array}{c}2 g+1 \\ g+1\end{array}\right)$, hence defines an invariant of $X(\mathbb{C})$. It follows from [9, Proposition 3.2] that the formula:

$$
n d(X)=-n \log \left\|\Lambda_{g}\right\|=-4 g^{2} r \log (2 \pi)-g \log \left\|\Delta_{g}\right\|(X)
$$

holds.

The invariant $\chi(X)$ of $X$ is determined by $d(X)$ and the invariants $\varepsilon(X)$ and $\delta(X)$ which we discuss next. The invariant $\varepsilon(X)$ stems from [17 and is defined as follows. Let $K$ again be non-Archimedean. We keep the assumption that $X$ has semistable reduction over $K$. Let $R(X)$ be the reduction graph of $X$, let $\mu_{X}$ be the admissible measure on $R(X)$, let $K_{X}$ be the canonical divisor on $R(X)$, and let $g_{X}$ be the admissible Green's function on $R(X)$. Then:

$$
\varepsilon(X)=\int_{R(X)} g_{X}(x, x)\left((2 g-2) \mu_{X}+\delta_{K_{X}}\right) .
$$

If $K$ is Archimedean, one simply puts $\varepsilon(X)=0$.

As to $\delta(X)$, for $K$ non-Archimedean we put $\delta(X)=\sum_{i=0}^{\lfloor g / 2\rfloor} \delta_{i}(X)$, the number of singular points on the special fiber of the minimal regular model of $X$ over $K$; if $K$ equals $\mathbb{R}$ or $\mathbb{C}$ we put $\delta(X)=-4 g \log (2 \pi)+\delta_{F}(X)$ where $\delta_{F}(X)$ is the Faltings delta-invariant of $X(\mathbb{C})$ defined on [3, p. 402].

The invariant $\chi(X)$ is determined by the following equality:

$$
(2 g-2) \chi(X)=3 d(X)-(2 g+1)(\varepsilon(X)+\delta(X)) .
$$

Let $K$ be non-Archimedean. It is clear that $\chi(X)=0$ if $X$ has good reduction, since each of $d, \varepsilon$ and $\delta$ vanishes in this case. 
From [13] one can calculate $\chi(X)$ in the case that $g=2$, based on the classification of the semistable fiber types in genus 2 . We display the results in a table:

\begin{tabular}{|l|cccc|}
\hline Type & $d / 2$ & $\delta$ & $\varepsilon$ & $\chi$ \\
\hline$I$ & 0 & 0 & 0 & 0 \\
$I I(a)$ & $2 a$ & $a$ & $a$ & $a$ \\
$I I I(a)$ & $a$ & $a$ & $\frac{1}{6} a$ & $\frac{1}{12} a$ \\
$I V(a, b)$ & $2 a+b$ & $a+b$ & $a+\frac{1}{6} b$ & $a+\frac{1}{12} b$ \\
$V(a, b)$ & $a+b$ & $a+b$ & $\frac{1}{6}(a+b)$ & $\frac{1}{12}(a+b)$ \\
$V I(a, b, c)$ & $2 a+b+c$ & $a+b+c$ & $a+\frac{1}{6}(b+c)$ & $a+\frac{1}{12}(b+c)$ \\
$V I I(a, b, c)$ & $a+b+c$ & $a+b+c$ & $\frac{1}{6}(a+b+c)$ & $\frac{1}{12}(a+b+c)$ \\
& & & $+\frac{1}{6} \frac{a b c}{a b+b c+c a}$ & $-\frac{5}{12} \frac{a b c}{a b+b c+c a}$ \\
\hline
\end{tabular}

In the case $g \geq 3$ one has an effective lower bound for $\chi(X)$ which is strictly positive in the case of non-smooth reduction, by work of Yamaki [16]. We quote his result:

$\chi(X) \geq \frac{(2 g-5)}{24 g} \xi_{0}(X)+\sum_{j=1}^{\lfloor(g-1) / 2\rfloor} \frac{3 j(g-1-j)-g-2}{3 g} \xi_{j}(X)+\sum_{i=1}^{\lfloor g / 2\rfloor} \frac{2 i(g-i)}{g} \delta_{i}(X)$

if $g \geq 5$, and:

$$
\chi(X) \geq \frac{(2 g-5)}{24 g} \xi_{0}(X)+\sum_{j=1}^{\lfloor(g-1) / 2\rfloor} \frac{2 j(g-1-j)-1}{2 g} \xi_{j}(X)+\sum_{i=1}^{\lfloor g / 2\rfloor} \frac{2 i(g-i)}{g} \delta_{i}(X)
$$

for the cases $g=3,4$. The most difficult part of the proof lies in obtaining suitable upper bounds for the $\varepsilon$-invariant ranging over the reduction graphs of hyperelliptic curves of a fixed genus using combinatorial optimisation.

If $K$ is Archimedean, one easily gets an exact formula for $\chi(X)$ from (4.2) and (4.3). We state the result for completeness:

$$
\chi(X)=-\frac{8 g(2 g+1)}{2 g-2} \log (2 \pi)-\frac{3 g}{(2 g-2) n} \log \left\|\Delta_{g}\right\|(X)-\frac{2 g+1}{2 g-2} \delta_{F}(X) .
$$

If $K$ is a number field or the function field of a curve over a field $k_{0}$, and $X$ has semistable reduction over $K$, one has the following global formulas involving $d, \varepsilon, \delta$ and the places $v$ of $K$. Let $N v$ be the following local factors at $v$ : in the number field case and for $v$ non-Archimedean, put $N v=\# \kappa(v)$ with $\kappa(v)$ the residue field at $v$; for $v$ Archimedean put $\log N v=1$ if $v$ is real, and $\log N v=2$ if $v$ is complex. In the function field case, let $\log N v$ be the degree of $v$ over the base field $k_{0}$. Then first of all:

$$
(8 g+4) \operatorname{deg} \operatorname{det} R \pi_{*} \omega=\sum_{v} d\left(X_{v}\right) \log N v,
$$

where $\operatorname{deg} \operatorname{det} R \pi_{*} \omega$ is the geometric degree of $\operatorname{det} R \pi_{*} \omega$ in the function field case, and the non-normalised Faltings height of $X$ in the number field case. This follows directly from the definition of $d$. Next one has:

$$
(\omega, \omega)_{a}=(\omega, \omega)-\sum_{v} \varepsilon\left(X_{v}\right) \log N v
$$


by [18, Theorem 4.4], where $(\omega, \omega)_{a}$ and $(\omega, \omega)$ are the admissible and usual selfintersections of the relative dualising sheaf of $X$ over $K$, respectively. Finally:

$$
12 \operatorname{deg} \operatorname{det} R \pi_{*} \omega=(\omega, \omega)+\sum_{v} \delta\left(X_{v}\right) \log N v
$$

which is the Noether formula for $X$ over $S$, cf. [12, Théorème 2.5] for the number field case.

We easily find the formula:

$$
(\omega, \omega)_{a}=\frac{2 g-2}{2 g+1} \sum_{v} \chi\left(X_{v}\right) \log N v
$$

expressing $(\omega, \omega)_{a}$ in terms of the $\chi\left(X_{v}\right)$. From the results of Moriwaki and Yamaki mentioned above one gets an effective version of the Bogomolov conjecture for $X$, if $K$ is a function field.

\section{INTRINSIC APPROACH TO $\chi$}

In this section we provide an alternative approach to $\chi$. We construct a canonical non-zero rational section $q$ of the line bundle $(2 g+1)\langle\omega, \omega\rangle$ on the base of a generically smooth semistable hyperelliptic curve of genus $g$, and show that $\chi$ is essentially the $-\log$ of the admissible norm of $q$.

A crucial ingredient of our approach is the arithmetic of the symmetric discriminants of a hyperelliptic curve. These are intimately related to the curve's symmetric roots, see [4, Section 2] for a discussion. The definition is as follows. Let $\kappa$ be a field not of characteristic 2 and let $X$ be a hyperelliptic curve of genus $g \geq 2$ over $\kappa$. Let $\bar{\kappa}$ be a separable algebraic closure of $\kappa$, and let $w_{1}, \ldots, w_{2 g+2}$ be the Weierstrass points of $X \otimes \bar{\kappa}$. Let $\left(w_{i}, w_{j}\right)$ be a pair of these. We have well-defined sets of symmetric roots $\left\{\ell_{i j k}\right\}_{k \neq i, j}^{\zeta}$ in $\bar{\kappa}$ associated to $i, j$, parametrised by the elements $\zeta$ of $\mu_{2 g}$. The $\zeta$ 's give rise to a set of symmetric equations:

$$
\mathcal{C}_{i j}^{\zeta}: y^{2}=x \prod_{k \neq i, j}\left(x-\ell_{i j k}\right)
$$

for $X$ over $\bar{\kappa}$, parametrised by $\zeta$. The discriminant:

$$
d_{i j}=\prod_{\substack{r, s \neq i, j \\ r \neq s}}\left(\ell_{i j r}-\ell_{i j s}\right)
$$

of a $\mathcal{C}_{i j}^{\zeta}$ is independent of the choice of $\zeta$ and is called the symmetric discriminant of the pair $\left(w_{i}, w_{j}\right)$. It is a well-defined element of the field of definition inside $\bar{\kappa}$ of $\left(w_{i}, w_{j}\right)$. A small calculation shows that the formula:

$$
\frac{d_{i k}}{d_{j k}}=-\ell_{i j k}^{2 g(2 g+1)}
$$

holds in $\bar{\kappa}$ for all $k \neq i, j$, allowing us to compute suitable powers of the symmetric roots of $X$ from the symmetric discriminants of $X$.

We start our construction of the rational section $q$ with the case of a smooth hyperelliptic curve $\pi: X \rightarrow S$ of genus $g$ where $S$ is a scheme whose generic points are not of characteristic 2. By [10, Proposition 7.3] there exists a faithfully flat morphism $S^{\prime} \rightarrow S$ such that the smooth hyperelliptic curve $X \times_{S} S^{\prime} \rightarrow S^{\prime}$ has $2 g+2$ sections $W_{1}, \ldots, W_{2 g+2}$ invariant for the hyperelliptic involution. Let $\left(W_{i}, W_{k}\right)$ be a pair of these. As we saw in Section 2 there exists a line bundle $Q_{i k}$ on $S^{\prime}$ associated 
to $\left(W_{i}, W_{k}\right)$ together with a canonical non-zero rational section $q_{i k}$ of $Q_{i k}$. We can and will view $q_{i k}$ as a rational section of $\langle\omega, \omega\rangle$ on $S^{\prime}$, by Proposition 2.1.

Let $d_{i k}$ be the symmetric discriminant of $\left(W_{i}, W_{k}\right)$, viewed as a rational function on $S^{\prime}$. We define:

$$
q=\left(2^{4 g} d_{i k}\right)^{g-1} \cdot q_{i k}^{\otimes 2 g+1},
$$

viewed as a rational section of $(2 g+1)\langle\omega, \omega\rangle$ on $S^{\prime}$.

Lemma 5.1. The rational section $q$ of $(2 g+1)\langle\omega, \omega\rangle$ is independent of the choice of $\left(W_{i}, W_{k}\right)$, and descends to a canonical rational section of $(2 g+1)\langle\omega, \omega\rangle$ on $S$.

Proof. To see this, first fix an index $k$ and consider the sections $\left(2^{4 g} d_{i k}\right)^{g-1} \cdot q_{i k}^{\otimes 2 g+1}$ and $\left(2^{4 g} d_{j k}\right)^{g-1} \cdot q_{j k}^{\otimes 2 g+1}$ for $i, j \neq k$. According to equation (5.1) we have:

$$
\frac{d_{i k}}{d_{j k}}=-\ell_{i j k}^{2 g(2 g+1)}
$$

whereas by Theorem 3.1 we have:

$$
q_{i k}^{-1} \otimes q_{j k}=\left(-\ell_{i j k}^{2 g}\right)^{g-1} .
$$

It follows that the $\left(2^{4 g} d_{i k}\right)^{g-1} \cdot q_{i k}^{\otimes 2 g+1}$ are mutually equal, where $i$ runs over the indices different from $k$. By symmetry considerations we can vary $k$ as well and the independence of $q$ on the choice of $(i, k)$ follows. By faithfully flat descent, see [14, Exposé VIII, Théorème 1.1], we obtain that $q$ comes from the base $S$.

Again let $\mathcal{I}_{g}$ be the moduli stack of smooth hyperelliptic curves of genus $g$ over $\mathbb{Z}$. By Lemma 5.1 we have $q$ as a canonical rational section of the line bundle $(2 g+1)\langle\omega, \omega\rangle$ on $\mathcal{I}_{g}$, and by pullback we obtain $q$ on the base of any smooth hyperelliptic curve. Even better, by extension we get $q$ as a rational section on the base of any generically smooth hyperelliptic curve. We isolate this result in a theorem. Let $S$ be an integral scheme, let $\pi: X \rightarrow S$ be a generically smooth hyperelliptic curve of genus $g \geq 2$ over $S$, and let $\omega$ be the relative dualising sheaf of $\pi$.

Theorem 5.2. The line bundle $(2 g+1)\langle\omega, \omega\rangle$ on $S$ has a canonical rational section $q$. If $S$ does not have generic characteristic equal to 2 , then $q$ is given by equation (5.2). The formation of $q$ is compatible with dominant base change.

The next result yields Theorems A and B as an immediate consequence.

Theorem 5.3. Assume that $S$ is a normal integral scheme.

(i) the rational section $q$ of $(2 g+1)\langle\omega, \omega\rangle$ is in fact a global section, with no zeroes if $\pi$ is smooth.

Let $K$ be either a complete discrete valuation field or let $K$ equal $\mathbb{R}$ or $\mathbb{C}$. Let $\bar{K}$ be an algebraic closure of $K$ and assume that $S=\operatorname{Spec}(\bar{K})$. Let $|\cdot|_{a}$ be the admissible norm on $(2 g+1)\langle\omega, \omega\rangle$. Then:

(ii) the formula:

$$
-\log |q|_{a}=(2 g-2) \chi(X)
$$

holds; 
(iii) if $K$ does not have characteristic 2 , and $w_{1}, \ldots, w_{2 g+2}$ on $X \otimes \bar{K}$ are the Weierstrass points of $X$, then the formula:

$$
-\log |q|_{a}=-4 g(g-1)\left(\log |2|+\sum_{k \neq i}\left(w_{i}, w_{k}\right)_{a}\right)
$$

holds, for all $i=1, \ldots, 2 g+2$, where $(,)_{a}$ denotes Zhang's admissible pairing on $\operatorname{Div}(X \otimes \bar{K})$.

Proof. To prove that $q$ has no zeroes if $\pi$ is smooth, note that it suffices to prove this in the case of the tautological curve over $\mathcal{I}_{g}$. We have that $\mathcal{I}_{g}$ is normal, as $\mathcal{I}_{g} \rightarrow \operatorname{Spec}(\mathbb{Z})$ is smooth by [8, Theorem 3]. Thus it is sufficient to prove the statement for the case of a smooth hyperelliptic curve $\pi: X \rightarrow S$ where $S$ is the spectrum of a discrete valuation ring $R$ of characteristic zero. We can and will assume that all Weierstrass points of the generic fiber of $\pi$ are rational, hence extend to sections $W_{1}, \ldots, W_{2 g+2}$ of $\pi$. Let $\nu(\cdot)$ denote order of vanishing along the closed point of $S$, and fix an index $i$. It is not hard to check the formula $\prod_{k \neq i} d_{i k}=1$ for the symmetric discriminants. This gives us, directly from the definition of $q$ :

$$
\nu(q)=4 g(g-1) \nu(2)+\sum_{k \neq i} \nu\left(q_{i k}\right) .
$$

Hence:

$$
\nu(q)=4 g(g-1)\left(\nu(2)-\sum_{k \neq i}\left(W_{i}, W_{k}\right)\right),
$$

where $($,$) denotes intersection product on \operatorname{Div}(X)$. Note that the $V$ 's are empty. If the residue characteristic of $R$ is not equal to 2 we immediately obtain the vanishing of $\nu(q)$ since $W_{i}$ is disjoint from each $W_{k}$. So assume that the residue characteristic of $R$ is equal to 2. It follows from the proof of Proposition 3.2 that:

$$
2 \sum_{k \neq i}\left(W_{i}, W_{k}\right)=\nu(4) .
$$

We see that $\nu(q)$ vanishes in this case as well. This proves the second half of (i).

Now let as above $\lambda=\operatorname{det} R \pi_{*} \omega$ be the Hodge bundle on $\overline{\mathcal{I}}_{g}$, the stack-theoretic closure of $\mathcal{I}_{g}$ in $\overline{\mathcal{M}}_{g}$, and let $\delta$ be the line bundle associated to the restriction of the boundary divisor of $\overline{\mathcal{M}}_{g}$ to $\overline{\mathcal{I}}_{g}$. By [12, Théorème 2.1] there exists an isomorphism:

$$
\mu: 3(8 g+4) \lambda-(2 g+1) \delta \stackrel{\cong}{\rightrightarrows}(2 g+1)\langle\omega, \omega\rangle
$$

of line bundles on $\overline{\mathcal{I}}_{g}$. On the left hand side one has a canonical non-zero rational section $\Lambda_{g}^{\otimes 3} \otimes \delta^{\otimes-(2 g+1)}$, and on the right hand side one has the canonical rational section $q$. We claim that under $\mu$, these two rational sections are identified, up to a sign. Indeed, the rational section $\Lambda_{g}^{\otimes 3} \otimes \delta^{\otimes-(2 g+1)}$ restricts to the global section $\Lambda_{g}^{\otimes 3}$ of $3(8 g+4) \lambda$ over $\mathcal{I}_{g}$ which is nowhere vanishing. By the second half of (i) we have that $q$ is nowhere vanishing as well, and hence, over $\mathcal{I}_{g}$, the image of $\Lambda_{g}^{\otimes 3} \otimes \delta^{\otimes-(2 g+1)}$ under $\mu$ differs from $q$ by an invertible regular function. It is stated in [5, Lemma 7.3] that such a function is either +1 or -1 . The claim follows.

The first half of (i) and statement (ii) follow from the fact that $\Lambda_{g}^{\otimes 3} \otimes \delta^{\otimes-(2 g+1)}$ and $q$ are identified, up to sign. As to the first half of statement (i), from the Cornalba-Harris equality (4.1) we see that, if $S$ is normal, the rational section 
$\Lambda_{g}^{\otimes 3} \otimes \delta^{\otimes-(2 g+1)}$ of $3(8 g+4) \lambda-(2 g+1) \delta$ is in fact global, as it gives rise to an effective Cartier divisor on $S$. It follows that $q$ is global, too.

As to (ii), first take $K$ to be a complete discrete valuation field. We may assume that $X$ has semistable reduction over $K$. Let $R$ be the valuation ring of $K$ and let $\mathcal{X} \rightarrow \operatorname{Spec}(R)$ be the regular minimal model of $X$. As above we denote by $\nu(\cdot)$ order of vanishing along the closed point of $\operatorname{Spec}(R)$. We have:

$$
\nu(q)=3 \nu\left(\Lambda_{g}\right)-(2 g+1) \nu(\delta)=3 d(X)-(2 g+1) \delta(X) .
$$

On the other hand, by [17, Theorem 4.4] we have:

$$
\nu(q)=-\log |q|_{a}+(2 g+1) \varepsilon(X),
$$

and indeed the formula:

$$
-\log |q|_{a}=(2 g-2) \chi(X)
$$

drops out.

Next let $K$ be equal to $\mathbb{R}$ or $\mathbb{C}$. By [12, Théorème 2.2] the isomorphism $\mu$, restricted to $\operatorname{Spec}(\bar{K})$, has admissible norm $\mathrm{e}^{(2 g+1) \delta(X)}$. It follows that:

$$
-\log |q|_{a}=-3 \log \left\|\Lambda_{g}\right\|(X)-(2 g+1) \delta(X)=(2 g-2) \chi(X)
$$

in this case as well.

The formula in (iii) follows directly from the definition of $q$. Fix an index $i$. Under the assumptions of (iii) we have:

$$
-\log |q|_{a}=-\log \left|\left(2^{4 g} d_{i k}\right)^{g-1} \cdot q_{i k}^{\otimes 2 g+1}\right|_{a}
$$

for all $k \neq i$, hence, using the identity $\prod_{k \neq i} d_{i k}=1$ once more:

$$
-\log |q|_{a}=-4 g(g-1) \log |2|-\sum_{k \neq i} \log \left|q_{i k}\right|_{a} .
$$

We find:

$$
-\log |q|_{a}=-4 g(g-1)\left(\log |2|+\sum_{k \neq i}\left(w_{i}, w_{k}\right)_{a}\right)
$$

as required.

Remark 5.4. Let $K$ be a number field or the function field of a curve over a field which is not of characteristic 2. Let $X$ be a hyperelliptic curve over $K$ of genus $g \geq 2$. Assume that $X$ has semistable reduction over $K$, and that all Weierstrass points $w_{1}, \ldots, w_{2 g+2}$ of $X$ are rational over $K$. Let $(\omega, \omega)_{a}$ be the admissible selfintersection of the relative dualising sheaf of $X$, and fix an index $i$. From (iii) of the above theorem we obtain, by summing over all places of $K$, that:

$$
(2 g+1)(\omega, \omega)_{a}=-4 g(g-1) \sum_{k \neq i}\left(w_{i}, w_{k}\right)_{a},
$$

where $\left(w_{i}, w_{k}\right)_{a}$ is now the global admissible pairing of $w_{i}, w_{k}$ on $X$. We note that from the fact that the global $(,)_{a}$ restricts to minus the Néron-Tate pairing on degree-0 divisors [17, Section 5.4] one may actually infer the stronger identity:

$$
(\omega, \omega)_{a}=-4 g(g-1)\left(w_{i}, w_{k}\right)_{a}
$$

for any two indices $i, k$. It is not true, however, that the local intersections $\left(w_{i}, w_{k}\right)_{a}$ are in general independent of the choice of $i, k$. 
Remark 5.5. The combinatorial optimisation methods used in [16] to prove effective lower bounds for $\chi$ in the non-Archimedean case are quite complicated. It would be interesting to see whether the simple formula from Theorem B could be used to obtain good lower bounds for $\chi$ in an easier fashion. Note that the global section $q$ in the function field case in some sense "explains" the strict positivity of $(\omega, \omega)_{a}$ for non-isotrivial hyperelliptic fibrations.

Remark 5.6. Let $K$ be the fraction field of a discrete valuation ring $R$ of residue characteristic not equal to 2, and fix a separable algebraic closure $\bar{K}$ of $K$. The symmetric equations $\mathcal{C}_{i j}^{\zeta}$ of a hyperelliptic curve $X$ over $K$ have good properties, for example:

- if $X$ has potentially good reduction over $R$, then the coefficients of $\mathcal{C}_{i j}^{\zeta}$ in $\bar{K}$ are integral over $R$, and $\mathcal{C}_{i j}^{\zeta}$ has good reduction over $R\left[\mathcal{C}_{i j}^{\zeta}\right]$;

- if the coefficients of $\mathcal{C}_{i j}^{\zeta}$ in $\bar{K}$ are integral over $R$, then $\mathcal{C}_{i j}^{\zeta}$ is a minimal equation of $X$ over $R\left[\mathcal{C}_{i j}^{\zeta}\right]$.

For details and proofs of these facts we refer to [4, Section 2].

\section{Connection With Zhang's $\varphi$-invariant}

Let $K$ be a field which is either a number field or the function field of a curve over a field. Let $X$ be a smooth projective geometrically connected curve of genus $g \geq 2$ over $K$, and assume that $X$ has semistable reduction over $K$. In a recent paper [18] S.-W. Zhang introduced, for each place $v$ of $K$, a real-valued invariant $\varphi\left(X_{v}\right)$ of $X \otimes K_{v}$, as follows:

- if $v$ is a non-Archimedean place, then:

$$
\varphi\left(X_{v}\right)=-\frac{1}{4} \delta\left(X_{v}\right)+\frac{1}{4} \int_{R\left(X_{v}\right)} g_{v}(x, x)\left((10 g+2) \mu_{v}-\delta_{K_{X_{v}}}\right),
$$

where:

- $\delta\left(X_{v}\right)$ is the number of singular points on the special fiber of $X$ at $v$,

- $R\left(X_{v}\right)$ is the reduction graph of $X$ at $v$,

- $g_{v}$ is the Green's function for the admissible metric $\mu_{v}$ on $R\left(X_{v}\right)$,

- $K_{X_{v}}$ is the canonical divisor on $R\left(X_{v}\right)$.

In particular, $\varphi\left(X_{v}\right)=0$ if $X$ has good reduction at $v$;

- if $v$ is an Archimedean place, then:

$$
\varphi\left(X_{v}\right)=\sum_{\ell} \frac{2}{\lambda_{\ell}} \sum_{m, n=1}^{g}\left|\int_{X\left(\bar{K}_{v}\right)} \phi_{\ell} \omega_{m} \bar{\omega}_{n}\right|^{2},
$$

where $\phi_{\ell}$ are the non-constant normalised real eigenforms of the Arakelov Laplacian on the compact Riemann surface $X\left(\bar{K}_{v}\right)$, with eigenvalues $\lambda_{\ell}>0$, and $\left(\omega_{1}, \ldots, \omega_{g}\right)$ is an orthonormal basis for the Hermitian inner product $(\omega, \eta) \mapsto \frac{i}{2} \int_{X\left(\bar{K}_{v}\right)} \omega \bar{\eta}$ on the space of holomorphic differentials.

The adelic invariant $\varphi$ connects the admissible self-intersection of the relative dualising sheaf of $X$ and the height of the canonical Gross-Schoen cycle on $X^{3}$. 
Theorem 6.1 (Zhang [18]). Let $(\omega, \omega)_{a}$ be the admissible self-intersection of the relative dualising sheaf of $X$, and let $\left\langle\Delta_{\xi}, \Delta_{\xi}\right\rangle$ be the height of the canonical GrossSchoen cycle on $X^{3}$. Then:

$$
(\omega, \omega)_{a}=\frac{2 g-2}{2 g+1}\left(\left\langle\Delta_{\xi}, \Delta_{\xi}\right\rangle+\sum_{v} \varphi\left(X_{v}\right) \log N v\right),
$$

where $v$ runs over the places of $K$. Here the $N v$ are the local factors associated to $v$ as discussed in Section 4 above. If $X$ is hyperelliptic, then $\left\langle\Delta_{\xi}, \Delta_{\xi}\right\rangle=0$, and hence the formula:

$$
(\omega, \omega)_{a}=\frac{2 g-2}{2 g+1} \sum_{v} \varphi\left(X_{v}\right) \log N v
$$

holds.

The invariant $\varphi$ arises in a natural way as an adelic intersection number on the self-product $X^{2}$ of $X$, cf. [18, Theorem 2.5.1]. Note the striking resemblance of $\varphi$ with $\chi$, for hyperelliptic curves: like $\varphi$, the invariant $\chi$ vanishes at each nonArchimedean place of good reduction, and one has (6.1) with $\chi$ instead of $\varphi$, see formula (4.4).

The canonical nature of both $\varphi$ and $\chi$ leads us to make the following:

Conjecture 6.2. Assume that $X$ is hyperelliptic. Then $\varphi\left(X_{v}\right)=\chi\left(X_{v}\right)$ for each place $v$ of $K$.

This conjecture has the following interesting corollary. Assume that $K$ is a number field. Let $\pi: \mathcal{X} \rightarrow S$ be the regular minimal model of $X$ over the ring of integers of $K$. Then $\pi$ is a generically smooth semistable hyperelliptic curve. By Theorem 5.3(i) we have $q$ as a canonical global section of the bundle $(2 g+1)\langle\omega, \omega\rangle$ on $S$, and by (ii) of the same theorem $(2 g-2) \chi\left(X_{v}\right)=-\log |q|_{a}$ at each place $v$ of $K$. The latter quantity is, as we have seen from the results of Moriwaki and Yamaki, non-negative if $v$ is non-Archimedean.

Now consider the case that $v$ is an Archimedean place. It is clear from the definition of $\varphi$ given above that $\varphi\left(X_{v}\right) \geq 0$. But in fact one has $\varphi\left(X_{v}\right)>0$, as is explained in [18, Remark 2.5.1]. So, if $\varphi\left(X_{v}\right)=\chi\left(X_{v}\right)$ would hold, it follows that $-\log |q|_{a}$ is positive on the Archimedean places. In other words, the section $q$ is a small section of $(2 g+1)\langle\omega, \omega\rangle$. The Bogomolov conjecture, i.e. the statement that $(\omega, \omega)_{a}$ is strictly positive, would follow in a conceptual way by taking the admissible Arakelov degree of $q$.

In [6] we computed $\varphi$ in the case that $g=2$. A comparison of the outcome with the results mentioned in Section 4 above yields that Conjecture 6.2 is true if $g=2$. Thus we have an alternative proof of the Bogomolov conjecture for genus 2 curves over number fields.

\section{ACKNOWLEDGMENTS}

The author was supported by VENI grant 639.033.402 from the Netherlands Organisation for Scientific Research (NWO). He thanks the Max Planck Institut für Mathematik in Bonn for its hospitality during a visit. 


\section{REFERENCES}

[1] S. Bosch, Formelle Standardmodelle hyperelliptischer Kurven. Math. Ann. 251 (1980), no. 1, 19-42. MR583822 (82b:14018)

[2] J.-B. Bost, J.-F. Mestre, L. Moret-Bailly, Sur le calcul explicite des "classes de Chern" des surfaces arithmétiques de genre 2. Séminaire sur les Pinceaux de Courbes Elliptiques (Paris, 1988). Astérisque No. 183 (1990), 69-105. MR1065156 (92g:14018b)

[3] G. Faltings, Calculus on arithmetic surfaces. Ann. of Math. 119 (1984), no. 2, 387-424. MR.740897 (86e:14009)

[4] J. Guàrdia, Jacobian Nullwerte, periods and symmetric equations for hyperelliptic curves. Ann. Inst. Fourier (Grenoble) 57 (2007), no. 4, 1253-1283. MR2339331(2008g:11105)

[5] R. de Jong, Explicit Mumford isomorphism for hyperelliptic curves. J. Pure Appl. Algebra 208 (2007), no. 1, 1-14. MR2269824 (2007k:14046)

[6] R. de Jong, Admissible constants for genus 2 curves. Bulletin of the LMS 42 (2010), 405-411. MR2651934

[7] I. Kausz, A discriminant and an upper bound for $\omega^{2}$ for hyperelliptic arithmetic surfaces. Compositio Math. 115 (1999), no. 1, 37-69. MR.1671741(2000e:14033)

[8] O.A. Laudal and K. Lønsted, Deformations of curves. I. Moduli for hyperelliptic curves. Algebraic geometry (Proc. Sympos., Univ. Troms $\varnothing$, Troms $\varnothing, 1977)$ 150-167, Lecture Notes in Mathematics 687, Springer, Berlin, 1978. MR.527233 (80g:14027)

[9] P. Lockhart, On the discriminant of a hyperelliptic curve. Trans. Amer. Math. Soc. 342 (1994), no. 2, 729-752. MR.1195511 (94f:11054)

[10] K. Lønsted, S.L. Kleiman, Basics on families of hyperelliptic curves. Compositio Math. 38 (1979), no. 1, 83-111. MR.523266 (80g:14028)

[11] S. Maugeais, Relèvement des revêtements p-cycliques des courbes rationnelles semi-stables. Math. Ann. 327 (2003), no. 2, 365-393. MR2015076 (2004j:14031)

[12] L. Moret-Bailly, La formule de Noether pour les surfaces arithmétiques. Invent. Math. 98 (1989), no. 3, 491-498. MR1022303 (91h:14023)

[13] A. Moriwaki, Bogomolov conjecture for curves of genus 2 over function fields. J. Math. Kyoto Univ. 36 (1996), no. 4, 687-695. MR1443744 (98e:14029)

[14] Séminaire de géométrie algébrique du Bois Marie 1960-61 I: Revêtements étales et groupe fondamental. Dirigé par A. Grothendieck. Documents Mathématiques 3, Société Mathématique de France 2003. MR2017446 (2004g:14017)

[15] K. Yamaki, Cornalba-Harris equality for semistable hyperelliptic curves in positive characteristic. Asian J. Math. 8 (2004), no. 3, 409-426. MR2129243 (2005j:14039)

[16] K. Yamaki, Effective calculation of the geometric height and the Bogomolov conjecture for hyperelliptic curves over function fields. J. Math. Kyoto Univ. 48 (2008), no. 2, 401-443. MR2436745 (2009h:14042)

[17] S.-W. Zhang, Admissible pairing on a curve. Invent. Math. 112 (1993), no. 1, 171-193. MR.1207481 (94h:14023)

[18] S.-W. Zhang, Gross-Schoen cycles and dualising sheaves. Invent. Math. 179 (2010), 1-73. MR2563759

Mathematical Institute, University of Leiden, P.O. Box 9512, 2300 RA Leiden, The NETHERLANDS

E-mail address: rdejong@math.leidenuniv.nl 